\title{
Intensity variations inside supergranules
}

\author{
N. Meunier ${ }^{1}$, R. Tkaczuk ${ }^{2}$, and Th. Roudier ${ }^{1}$ \\ ${ }^{1}$ Laboratoire d'Astrophysique de l'Observatoire Midi-Pyrénées, Université Paul Sabatier Toulouse III, CNRS, 57 avenue d'Azereix, \\ BP 826, 65008 Tarbes Cedex, France \\ e-mail: [nadege.meunier; thierry.roudier]@ast.obs-mip.fr \\ 2 Laboratoire d'Astrophysique de l'Observatoire Midi-Pyrénées, Université Paul Sabatier Toulouse III, CNRS, \\ 14 avenue Edouard Belin, 31000 Toulouse, France \\ e-mail: ruben.tkaczuk@ast.obs-mip.fr
}

Received 29 August 2006 / Accepted 24 October 2006

\section{ABSTRACT}

\begin{abstract}
Context. The convective origin of supergranulation is highly controversial. Past measurements of intensity variations inside supergranules have often been influenced by the brightness enhancement at the cell boundaries due to the magnetic network.

Aims. We conduct a precise determination of intensity variations inside supergranules.

Methods. We determine the supergranule cell boundary from smoothed divergence maps derived from horizontal flow maps. We derive these flow maps from intensity maps obtained by MDI/SOHO in high resolution mode. We discuss the different possible approaches to take into account the influence of the magnetic field which can be used to determine the intensity variations inside supergranules.

Results. We observe a significant decrease of the intensity from the center to the boundary of supergranules. We also obtain additional clues from the behavior of the maximum intensities and minimum intensities around each pixel, which are related to granules and intergranules: the maximum intensity decreases from center to boundary, while the minimum intensity is constant or increases depending how restrictive the selection is. The difference between intensity profiles versus divergence and relative distance to cell center also provides complementary information. The corresponding temperature differences between cell center and boundary are in the range $0.8-2.8 \mathrm{~K}$. The intensity enhancement (for the magnetic network) or deficit (for intranetwork fields) depends on the localisation inside the cell.

Conclusions. It is the first time that such a detailed analysis of intensity variations inside supergranulation is performed. Our results are compatible with a convective origin of supergranulation, as the intensity decreases toward the boundary of the cells. However, new simulations of supergranulation are necessary to verify whether the compared behavior of granule and intergranule intensity variations is in close agreement with convection.
\end{abstract}

Key words. Sun: granulation - Sun: photosphere - Sun: general

\section{Introduction}

Supergranulation was discovered by Hart (1954). It has long been thought to be due to convection (Simon \& Leighton 1964; van der Borght 1979). Such an interpretation has however been questioned, in particular by Rieutord et al. (2000) and Rast (2003b). One clue to understand the origin of supergranulation would be the determination of intensity variations inside supergranules, as it is expected that a convective cell will exhibit a lower temperature at the boundary of the cell compared to the cell center. Most studies of intensity variations across supergranules found an enhancement at the boundary due to the presence of the magnetic network (Beckers 1968; Frazier 1970; Foukal \& Fowler 1984; Lin \& Kuhn 1992). More recently however, Rast (2003a) found a small decrease in intensity from cell center to the boundary when keeping pixels associated with very low magnetic field only (keeping less than $2.5 \%$ of the pixels and down to $0.1 \%$ ).

In this work, we study the variation of intensities inside supergranules in detail. We determine the intensity variations as a function of the smoothed divergence and as a function of the position inside the cell. We study not only the intensity at a given pixel in the images, but also its magnetic environment, in order to make a better diagnosis. The data processing is described in Sect. 2. We also discuss the different possible approaches to take into account the influence of the magnetic field on intensity variations. The results obtained with different pixel selections are described in Sect. 3 and discussed in Sect. 4.

\section{Data processing}

\subsection{The MDI data set}

We use two time series of high resolution MDI/SOHO observations (Scherrer et al. 1995) obtained with a 1 min temporal cadence: $46 \mathrm{~h}$ in January 1997 and $15 \mathrm{~h}$ in March 1997. We use intensity maps (continuum of the Ni $6768 \AA$ line) and magnetograms. These maps cover $\sim 620^{\prime \prime} \times 303^{\prime \prime}$ and the pixel size is 0.6054 arcsec. The observed regions are very quiet, except for the presence of a pore with active network surrounding it in the January time series. The area around the pore is eliminated from the analysis.

The rest of this section describes the data analysis. The steps described in Sect. 2.2 are similar to those described in detail in Meunier et al. (2007), except for the additionnal intensity maps. 


\subsection{Map computation}

\subsubsection{Intensity maps}

We consider 1-h long sets of intensity maps. In each set, the time cadence is $1 \mathrm{~min}$. Large-scale intensity gradients are present in MDI intensity maps, due to the center-to-limb darkening and to residuals from the flat-field correction. A first correction is therefore applied to the maps (see Meunier et al. 2007, for more details). Over each 1 -h period, the 60 corrected intensity maps are then carefully aligned with the image at the center of the 1-h set.

In the following, we will present results obtained using 5 successive intensity maps (therefore covering $4 \mathrm{~min}$ ) averaged together. The resulting map is denoted $I$ hereafter. Most of the solar oscillation signal is then removed. However, similar computations can be done with the filtered intensity maps (see Sect. 2.2.2), and they lead to similar results, which shows their robustness. For clarity, these results are therefore not presented here. There is a very large intensity dispersion in the intensity maps, much larger than the effect we wish to detect. The dispersion is however intrinsic to the solar photosphere as it is due to solar granulation. One could smooth them out before the analysis, for example using a spatial smoothing. However, this approach is not adequate because it would mix the contributions from the magnetic field coming from different pixels with no way to sort them out afterward.

For a better diagnosis of the intensity variations, we compute additional intensity maps to clarify the results obtained with the intensity $I$. For each pixel of the intensity map, we compute the minimum intensity $I_{\min }$ and the maximum intensity $I_{\max }$ over a $7 \times 7$ pixel box around the original pixel. The size of the box has been chosen so that it covers a typical granule. These intensities give some information on the intensity inside the granules themselves and in the corresponding intergranules. Of course, due to the limited spatial resolution of the instrument, the information is not complete as the intensities are smoothed in the original maps.

\subsubsection{Velocity maps}

The horizontal flow fields are computed using a Local Correlation Tracking method between two consecutive images in each 1-h intensity data set, after a k- $\omega$ filtering with a cut-off at $6 \mathrm{~km} \mathrm{~s}^{-1}$. These velocity maps are then averaged over each $1-\mathrm{h}$ period. This leads to 61 velocity maps aligned with the intensity maps.

\subsubsection{Divergence maps}

A divergence map is then computed for each velocity field map and moothed in the Fourier space using a 2D Gaussian with a $F W H M$ of $10.2 \mathrm{Mm}$. We refer to Meunier et al. (2007) for the choice of this spatial smoothing. It provides a supergranule cell size distribution in agreement with expected results (see Sect. 2.2.4).

\subsubsection{Cell determination}

In this paper, we use two ways of spatially characterizing the supergranular cells. First, we define the cells using the smoothed divergence maps, and then derive a normalized distance to the center of gravity of the cell. Second, we directly use the smoothed divergence defined in Sect. 2.2.3.

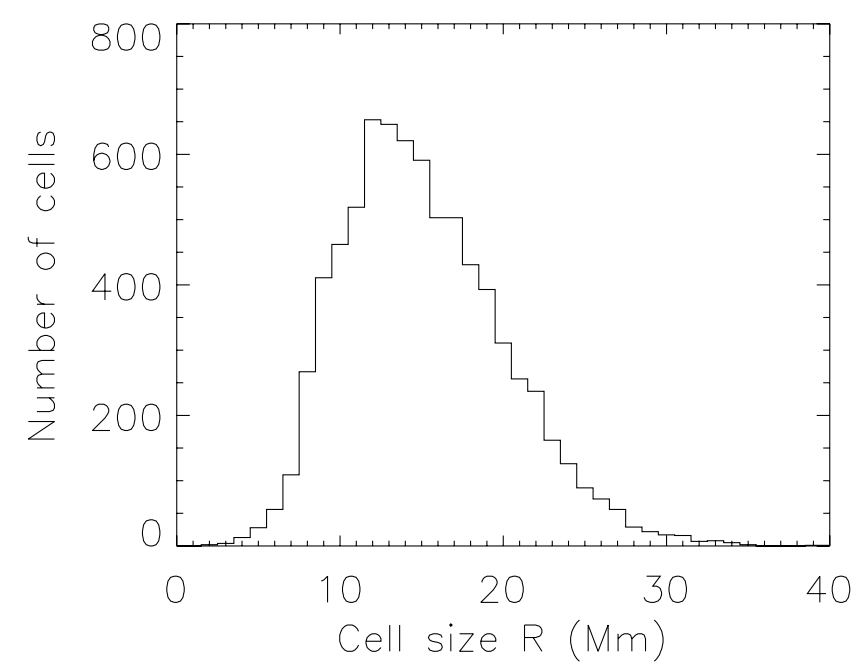

Fig. 1. Size distribution of the cell size $R$ in Mm. $R$ represents the maximum distance between the center of gravity of the cell and the boundary of the cell.

First, we define the boundary of the cells using a steepest descent method (see Hagenaar et al. 1997; Meunier et al. 2007). Then, for each pixel of the cell we compute the relative distance to the center of gravity of the cell. This relative distance to the cell center, hereafter $d_{\text {rel }}$, takes the value 0 at the center of gravity, and 1 for the pixels located at the boundary of the cell. The normalisation by cell size is very convenient when averaging the results from all cells. Figure 1 shows the distribution of the cell sizes $R$ for the 7629 cells, where $R$ is the maximum distance between the center of gravity and the cell boundary. The average value of $R$ is $15.6 \pm 0.1 \mathrm{Mm}$. It extends up to $40 \mathrm{Mm}$, but there are in fact only a few cells above $30 \mathrm{Mm}$.

A given divergence value does not correspond to the same location in the cell for different sizes (Meunier et al. 2007): for example, an intermediate value will correspond to the cell center in a small cell and to a position further from the cell center in a large cell, where the maximum divergence is much larger. As a consequence, we have also normalized the smoothed divergence using the following method. In each cell, the positive divergences are normalized by the maximum positive divergence in the cell. The same is done for the negative divergences (converging flows). This correction leads to a divergence $D^{\prime}$. We then consider $D_{\text {norm }}$ using the following formula :

$D_{\text {norm }}=\left(-D^{\prime}+1\right) \times 0.5$.

This normalized smoothed divergence $D_{\text {norm }}$ takes the value of 0 at the location of maximum positive divergence in the cell (diverging flows), 1 at the location of maximum negative divergence (converging flows) and 0.5 when the divergence is equal to zero. The results obtained can be easily averaged and compared to those obtained with $d_{\text {rel }}$. Furthermore, the maximum of $D_{\text {norm }}$ now corresponds to the maximum of $d_{\text {rel }}$, which also makes the comparison with $d_{\text {rel }}$ much easier to visualize.

Note that there is not a one to one comparison between the two methods (the correlation factor is 0.70 ), as there is a large dispersion. This is primarily due to the fact that the strongest positive divergences do not necessarily coincide with the center of gravity of the cell. Second, even if the strong convergences are located on the edge of the cell, many pixels of the boundary do not exhibit strong convergence as shown in Fig. 2. In all cases, 20 pixel-wide bands are removed at the egdes of the images to 

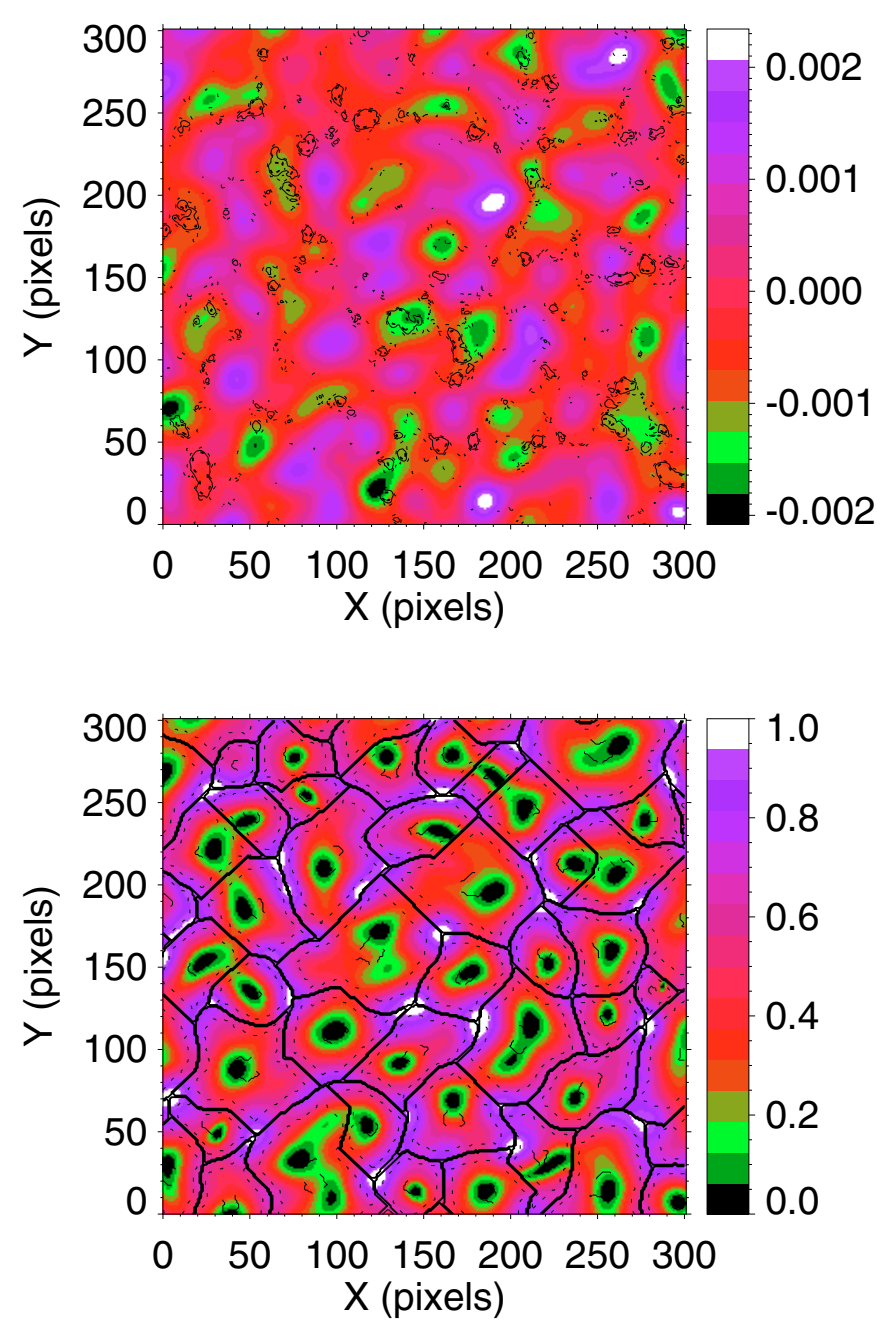

Fig. 2. Upper panel: smoothed divergence map (in $\mathrm{s}^{-1}$ ) for a $300 \times$ 300 pixels square. The line contours represent the magnetic field derived from the 1-hour averaged magnetogram at the levels of 10, 20 and $40 \mathrm{G}$ (i.e. the magnetic network). Lower panel: normalized divergence map $D_{\text {norm }}$ as color level. The solid line contours represent the cell boundaries. The dashed lines represent the contour of $d_{\text {rel }}$ at the 0.2 level, and the dotted line at the 0.8 level.

take into account the spatial smoothing. Cells overlapping these boundaries are also eliminated. Note that the results presented in this paper are very similar when considering the divergence before the normalization, or when considering another geometrical approach such as the distance to the center of gravity divided by the maximum size $R$ : the exact values are different but are of the same order of magnitude and as significant.

\subsection{How to determine the influence of the magnetic fields}

For each 1-h set, magnetograms are aligned with the image at the center of the set, and then averaged. Figure 2 also shows a subset from a smoothed divergence image. The magnetic network is superimposed on the divergence map, as well as cell boundaries and contours of $d_{\text {rel }}$. It shows that strong converging flows coincide well with the boundary, but there are many pixels of the boundary with almost absent converging flows. This magnetic field is actually magnetic flux in each pixel.
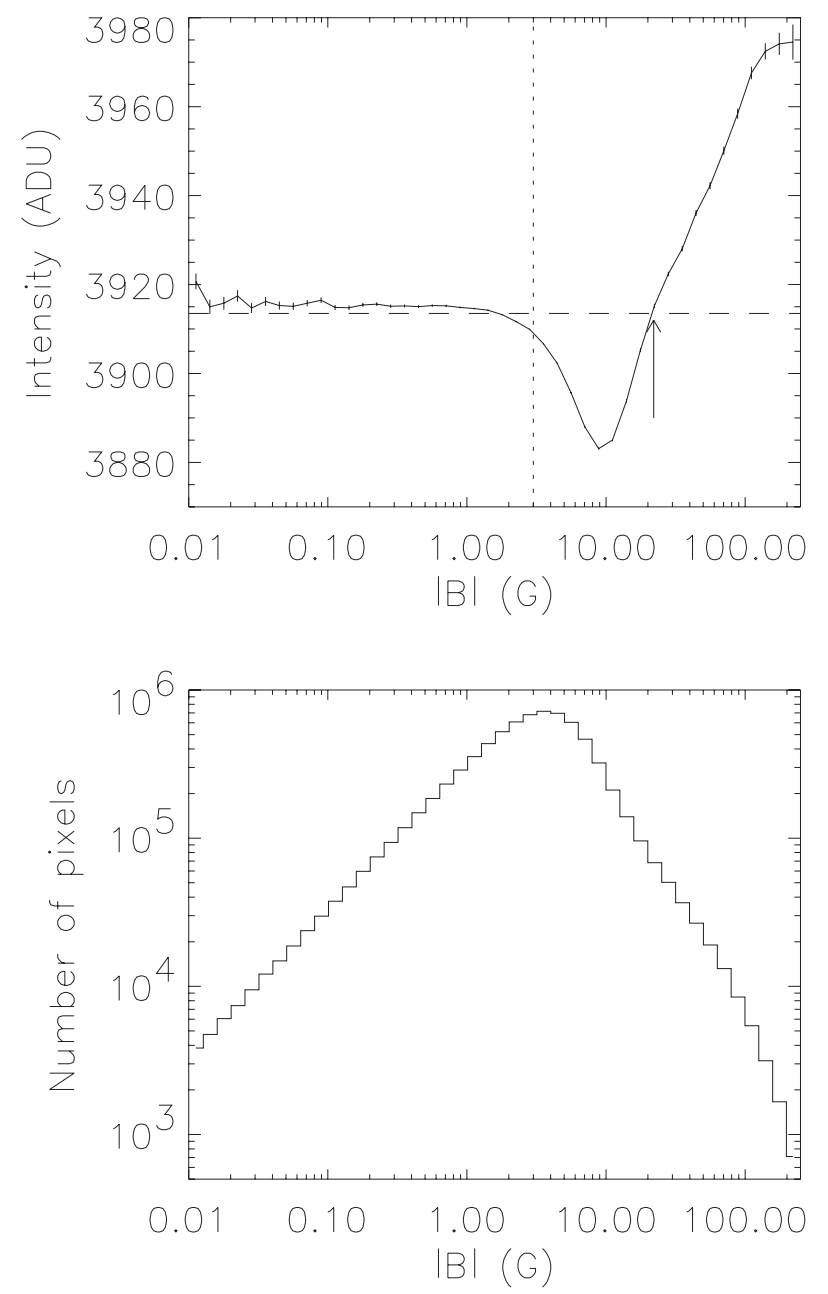

Fig. 3. Upper panel: intensity variations versus the absolute value of the magnetic field $|B|$, for the March 1997 time series. The horizontal dashed line represents the average intensity level for $|B|<3 \mathrm{G}$. The vertical dotted line, at $|B|=3 \mathrm{G}$, corresponds to the noise level. The arrow indicates the magnetic field value for which the intensity variation changes sign with respect to the low magnetic field intensity level. Lower panel: number of pixels versus the absolute value of the magnetic field $|B|$.

The presence of a magnetic field can contribute to intensity variations, in particular through unresolved bright points (Koutchmy \& Lebecq 1986; Title et al. 1987; Wang 1988; Title et al. 1992; Montagne et al. 1996). Figure 3 shows the variation of the intensity versus the magnetic field for the March 1997 time series. Below the noise level of $3 \mathrm{G}$ (defined at the $1 \sigma$ level in the 1-h averaged magnetograms), a constant intensity level, $I_{0}$, is observed. Above that threshold, two domains of magnetic fields are observed. In the range $3-20 \mathrm{G}$, the intensity is less than $I_{0}$, with a maximum difference to $I_{0}$ of $0.8 \%$ for $|B| \sim 10 \mathrm{G}$. This domain corresponds to weak field structures spread over the cells and is likely to be a component of the intranetwork magnetic field. For $|B|$ larger than $\sim 20 \mathrm{G}$, the intensity increases with $|B|$ and is larger than $I_{0}$, reaching values greater than $I_{0}$ by $1.5 \%$. This domain corresponds to magnetic network structures.

Several approaches are possible to eliminate the effects of $|B|$. We could correct the intensities, study the variations of the intensity with the magnetic field for several domains in the cells or select pixels for which the influence of the magnetic field is very small. These approaches are described and discussed below. 

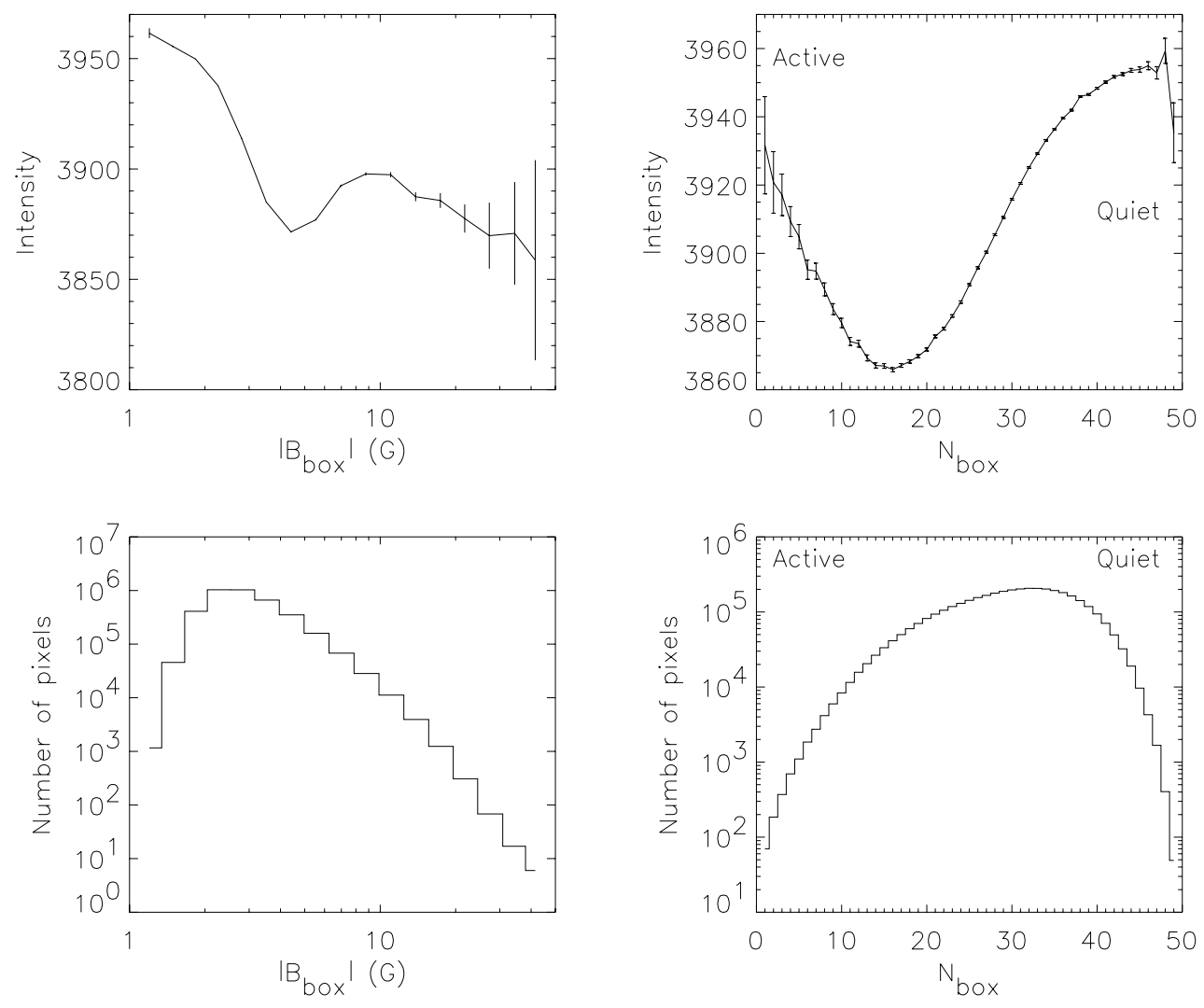

Fig. 4. Left panel: Intensity variations versus the absolute value of the magnetic field $\left|B_{\text {box }}\right|$ for $|B|<3 \mathrm{G}$ (upper panel) and number of pixels (lower panel), for the March 1997 time series. $\left|B_{\text {box }}\right|$ is the average magnetic field in a $7 \times 7$ pixels box around the current pixel. Left panel: same versus $N_{\text {box }}$, where $N_{\text {box }}$ is the number of pixels below $3 \mathrm{G}$ in a $7 \times 7$ pixels box around the current pixel.

\subsubsection{Intensity correction}

It should be possible to use the observation of the dependence of the intensity on the magnetic field to correct the original intensity and then to look at the spatial variations of the corrected intensities. The observed magnetic field dependence of the intensity is shown in Fig. 3. For example, if we note $I_{b}$, the intensity, as a function of $|B|$ as shown in Fig. 3 , the intensity $I$ could be corrected by computing $I+I_{0}-I_{b}$. The corrected intensity variations versus $d_{\text {rel }}$ or $D_{\text {norm }}$ for three domains of magnetic fields (namely $|B|<3 \mathrm{G}$, in the range 3-20 $\mathrm{G}$ and $>20 \mathrm{G}$ ) approach each other but they are not the same yet. For $|B|>20 \mathrm{G}$ in particular, they remain very different despite the correction.

This may be due to the fact that the intensity-magnetic field relationship does not depend only on $|B|$. We therefore define $N_{\text {box }}$ as the number of pixels with $|B|$ below the $3 \mathrm{G}$ threshold in a $7 \times 7$ pixel box around the current pixel. This size of the box was chosen to cover a typical granule as in Sect. 2.2.1. This variable will be used throughout the paper to characterize the influence of the magnetic environment on the intensity of a given pixel: pixels with a low $N_{\text {box }}$ value, despite corresponding to a very weak magnetic field $(|B|<3 \mathrm{G})$, may be surrounded by an active environment, while pixels with $N_{\text {box }}$ close to 49 are in very quiet regions. The average absolute magnetic field over the same box, hereafter $\left|B_{\text {box }}\right|$, is also computed and will be used to check the amplitude of the influence of the magnetic field in the environment of the current pixel. For small values of $N_{\text {box }}$, the average magnetic field $|B|$ is large, with an average $|B|$ and $\left|B_{\text {box }}\right|$ around $25 \mathrm{G}$ for $N_{\text {box }}$ close to 0 and around $8 \mathrm{G}$ for $N_{\text {box }}$ around 10. For large values of $N_{\text {box }}$, these values are lower than $2 \mathrm{G}$. The size of the magnetic structures defined by a $10 \mathrm{G}$ threshold can also help to interpret the significance of $\left|B_{\text {box }}\right|$ and $N_{\text {box }}$. We find that above 40, there is no overlap between the location of these pixels and the magnetic network.
We observe a large intensity variation versus $\left|B_{\text {box }}\right|$ and $N_{\text {box }}$ mostly due to the surroundings, even when we consider only pixels with a very low magnetic field, in this example $|B|<3 \mathrm{G}$, as shown in Fig. 4. For $|B|<3 \mathrm{G}$, the intensity is indeed higher for small values of $\left|B_{\text {box }}\right|$ and smaller for large values. On the other hand, the smaller intensities are observed for intermediate $N_{\text {box }}$, around 15 pixels. Intensity corrections taking into account this new complex dependence still lead to different dependences on the localisation for various $|B|$ domains. This should further complicate the correction to be performed on the intensity maps and we conclude that such a correction is too uncertain to allow the use of all pixels of the maps.

\subsubsection{Intensity dependence on the magnetic field across the cells}

A second possible approach is to compute the intensity variations versus $|B|$ for various locations in the cells. If there is an offset between the curves, then this may be a good indication of intensity variations across supergranules, and such a plot would also allow us to directly take into account the influence of the magnetic field. The result is shown in Fig. 5, where the intensity as a function of the magnetic field is shown separately for selections of pixels in strong divergences and convergences. The result is similar for pixels at cell center and at the cell boundary. The intensity deficit for $|B|$ in the range $3-20 \mathrm{G}$ has a smaller amplitude close to the cell boundary (or to regions of convergence), which suggests that weak field structures, likely to correspond to intranetwork magnetic fields, are associated with lower intensities than the surroundings, but that the intensities are higher closer to the cell boundary. It is the opposite for the high intensities observed for $|B|$ greater than $20 \mathrm{G}$, corresponding to the magnetic network: the network features are brighter close to the cell center than at the boundary, for a given magnetic field. This 


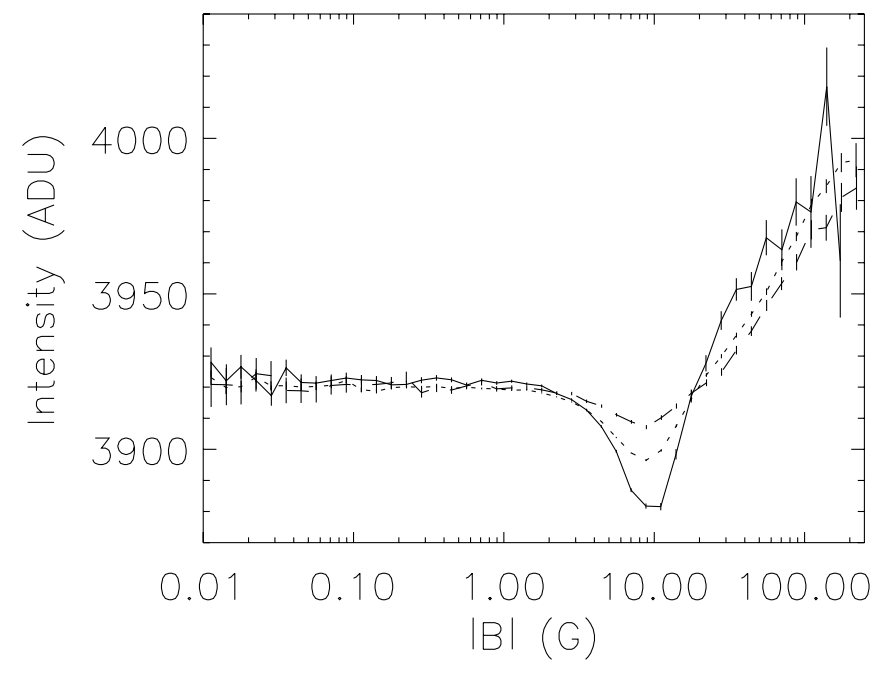

Fig. 5. Intensity variations versus the absolute value of the magnetic field $|B|$ for 3 ranges of $D_{\text {norm }}: D_{\text {norm }}$ lower than 0.2 (solid line), in the range $0.2-0.8$ (dotted line) and larger than 0.8 (dashed line).

is an important result, showing again that it is difficult to directly apply a correction to the intensity maps, as it is likely that the intensity properties for a given magnetic field vary with the localisation inside the cell.

When considering $|B|$ lower than $3 \mathrm{G}$, the variations are much smaller and we obtain a smaller intensity in convergences (compared to divergences) and at the cell boundary (compared to cell center), with a variation of the order of 1 ADU (Analog Digit Unit). This observation leads naturally to the approach described in the next section, which consists of selecting the pixels in the quietest regions.

\subsubsection{Pixel selection}

A third approach, which will be followed in the rest of this paper, is to select pixels for which the intrinsic intensity variations are very small. We consider pixels with $|B|$ below a threshold of $3 \mathrm{G}$ only, as most pixels above that threshold have a significant magnetic field and we are interested in extrapolating the intensity variations for $B=0$. To discriminate further, we also use the criterion $N_{\text {box }}$ defined in Sect. 2.3.1. Only pixels with $N_{\text {box }}$ above 7 are retained. Pixels with $N_{\text {box }}$ close to 49 are in very quiet regions and will be the most suitable for our analysis. We will study the dependence of the intensity variations on $N_{\text {box }}$ in order to identify the influence of the magnetic field on these intensity variations.

The noise level in the magnetograms varies over the field of view. In particular, there is a region located in the bottomright corner of the images with a significantly higher noise level. In practice, in this region, $N_{\text {box }}$ is always very low due to this noise, and almost all pixels in this region are eliminated from the analysis.

\subsection{Final parameters and errorbars}

We obtain a number of variables defined in each pixel of the maps where $|B|$ is below $3 \mathrm{G}$ and $N_{\text {box }}$ larger than 7 pixels:

- Intensity informations: $I, I_{\min }, I_{\max }$

- Cell property informations: $R, d_{\mathrm{rel}}, D_{\text {norm }}$

- Magnetic field informations: $|B|, N_{\text {box }},\left|B_{\text {box }}\right|$.
They are defined for slightly more than $10^{7}$ pixels, which covers $34 \%$ of the surface. The other pixels have a magnetic field above $3 \mathrm{G}$ or are too close to the edges of the images. All intensities are in ADU, i.e. the original CCD counts.

The correlation factor between 2 successive divergence maps is of the order of 0.6 . It falls to $\sim 0$ in $\sim 22$ hours. This means that the maps are not entirely independent from one hour to the next. In the following, the errorbars will be given by the standard deviations at the $1 \sigma$ level. This is not entirely correct due to this correlation and they could be overestimated by a factor of less than 2. On the other hand, the intensity maps are uncorrelated as over one hour the granulation pattern is completely different.

\section{Results}

Before studying the intensity variations over the selected pixels, we examine the influence of the magnetic field around the current pixel on the results. We apply a more severe selection in order to eliminate the influence of the magnetic field. The possible biases will be discussed.

\subsection{Influence of the magnetic environement}

We first study the intensity variations as a function of $N_{\text {box }}$, which characterizes the activity level in a granule-size region around the current pixel. The number of pixels as a function of $N_{\text {box }}$ was shown in Fig. 4 (lower-right panel) and peaks for $N_{\text {box }} \sim 33$. To characterize the intensity variations with $d_{\text {rel }}$ and $D_{\text {norm }}$, we compute the slope of the intensity as a function of $d_{\text {rel }}$ or $D_{\text {norm }}$. A negative slope means than the intensity is lower at the cell boundary. These slopes can be obtained for various pixel selections. Figure 6 shows the variation of the slope for the intensity $I$ as a function of $N_{\text {box }}$. The plot for $D_{\text {norm }}$ (upper panel) shows that the slope is close to 0 for small values of $N_{\text {box }}$, i.e. when the environment of the pixels associated with low magnetic fields is more active. However, the slope becomes negative at larger values $\left(N_{\text {box }}\right.$ above $\left.\sim 37\right)$. The result is similar for the variation with $d_{\text {rel }}$ (lower panel), although the change in sign appears at smaller values $\left(N_{\text {box }}\right.$ above $\left.\sim 23\right)$. Therefore there is a strong indication that when looking at the quietest regions, we observe a significantly lower intensity at the cell boundary.

The influence of the network magnetic field is expected to lead to an increase of the intensity at the cell boundary, i.e. where these strong magnetic fields are predominantly located. When considering the minimum and maximum intensities around each current pixel, defined by $I_{\min }$ and $I_{\max }$, it is possible to study the influence of the environment. The variations of $I_{\min }$ and $I_{\max }$, which are less dependant on the $3 \mathrm{G}$ threshold (as they can have larger values), show an even larger variation for intermediate values of $N_{\text {box }}$. In particular, the slope for $I_{\min }$ is positive, which may be due to an intensity increase in the intergranules due to the presence of activity there.

\subsection{Intensity variations in very quiet regions}

\subsubsection{Pixel selection}

In this section we study in more detail the intensity variations as a function of $D_{\text {norm }}$ and $d_{\text {rel }}$ for the quietest regions. We first consider the pixels where $N_{\text {box }}$ is larger than 40 . In that case, we are well in the range where the intensity variations are significant, with a negative slope. This leads to a selection of 755934 pixels, which represent $2.5 \%$ of the surface. 

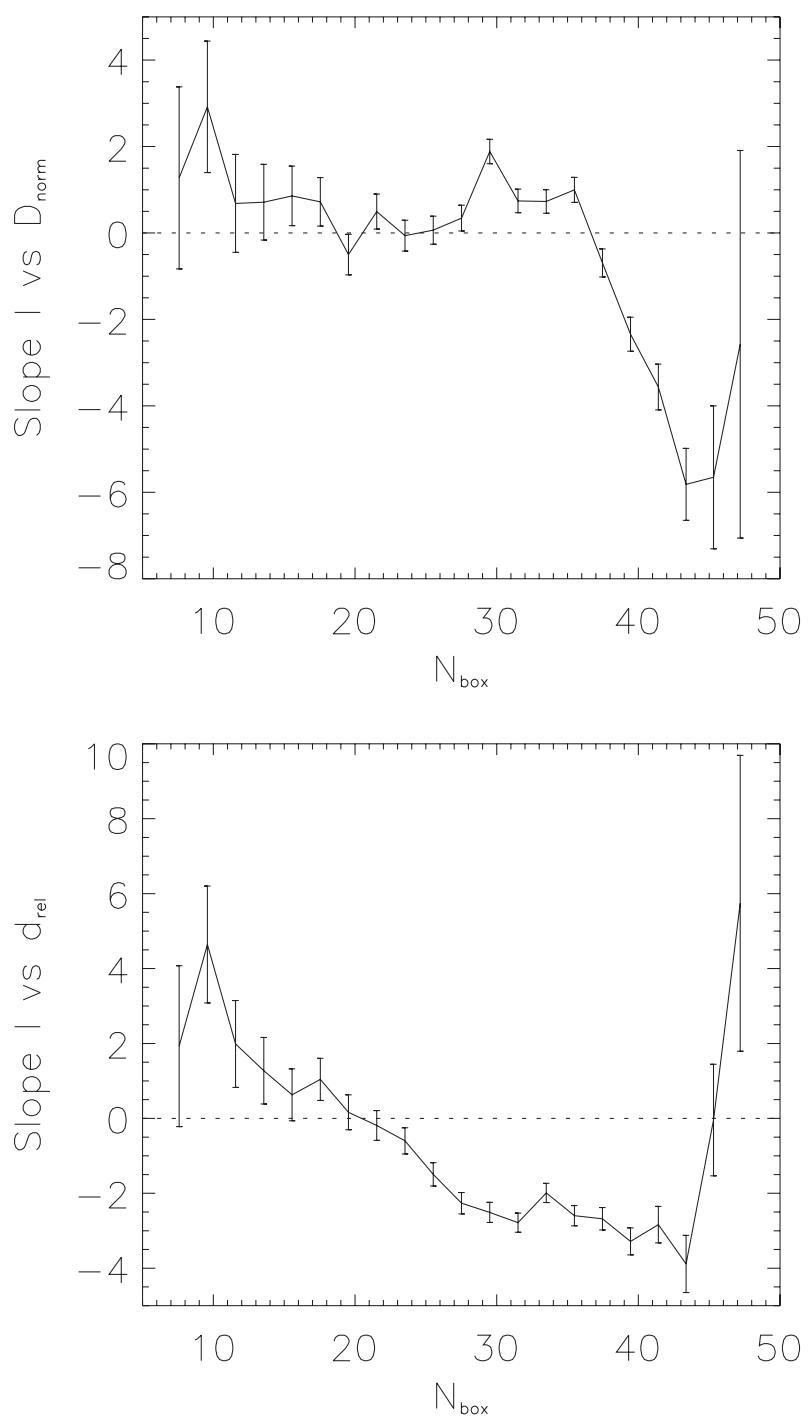

Fig. 6. Upper panel: slope of the intensity $I$ versus the normalized divergence $D_{\text {norm }}$, as a function of $N_{\text {box }}$, for $|B|<3 \mathrm{G}$. $N_{\text {box }}$ is the number of pixels below $3 \mathrm{G}$ in a $7 \times 7$ pixels box around the current pixel. Lower panel: same for the slope versus the relative distance to cell center $d_{\text {rel }}$.

Figure 7 shows the number of pixels versus $D_{\text {norm }}$ and $d_{\text {rel }}$. In the case of $D_{\text {norm }}$, there are more pixels for values smaller than 0.5 , i.e. the slopes will be more strongly influenced by this part of the cell. This is due to the fact that most pixels associated with strong magnetic fields are located in convergence regions. In the case of $d_{\text {rel }}$, it is the opposite and there are more pixels for values larger than 0.5 , so the slope will be more sensitive to the outer region of the cells. This is simply due to the definition of $d_{\text {rel }}$ : in a circular cell for example there will be many more pixels with $d_{\text {rel }}$ larger than 0.9 than smaller than 0.1 . Despite the low percentage of pixels kept for the analysis, they are widely distributed over the cells. Furthermore, pixels are not distributed randomly but tends to cover significant part of granules, with $80 \%$ of the pixels in structures larger than 10 pixels (about $2.5 \times 2.5$ arcsec). This will limit possible biases due to the preferential location with respect to granules and intergranules (see also Sect. 3.2.5). This low percentage also shows that there is a significant magnetic field almost everywhere on the surface. The decrease in number of pixels versus $d_{\text {rel }}$ and $D_{\text {norm }}$ at the boundary is also due to the presence of more magnetic flux there.
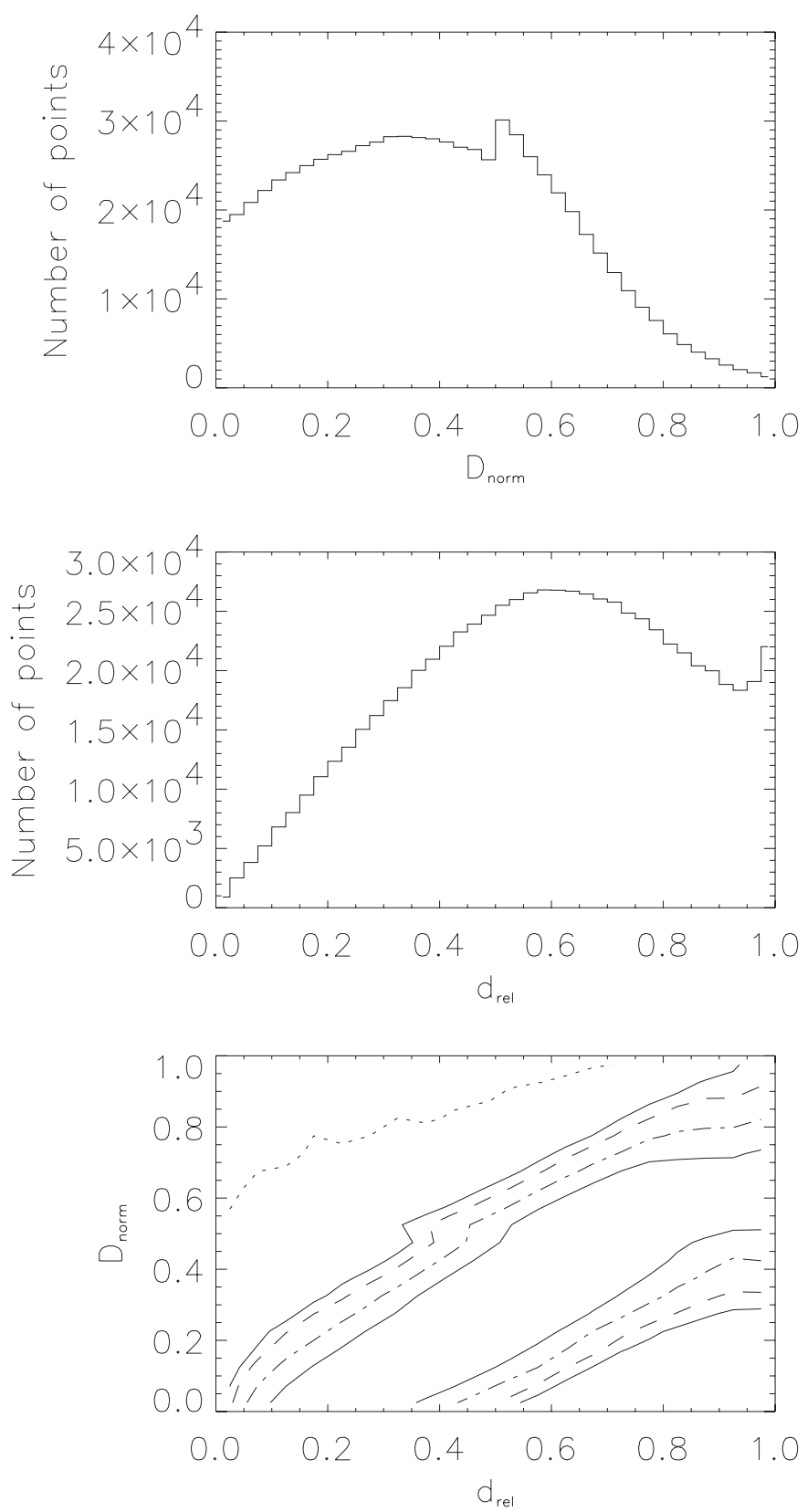

Fig. 7. Top panel: number of pixels versus $D_{\text {norm }}$ for $N_{\text {box }}$ larger than 40 and $|B|<3 \mathrm{G}$, where $N_{\text {box }}$ is the number of pixels below $3 \mathrm{G}$ in a $7 \times 7$ pixels box around the current pixel. Middle panel: number of pixels versus $d_{\text {rel }}$ for $N_{\text {box }}$ larger than 40 . Lower panel: number of pixels versus $D_{\text {norm }}$ and $d_{\text {rel }}$ for $N_{\text {box }}$ larger than 40 . Contours correspond to 10 pixels (dotted line), 900 pixels (solid line), 1300 pixels (dashed line), 2200 pixels (dotted-dashed line) and 3500 pixels (dot-dot-dot-dashed line).

Figure 7 also shows the 2D distribution of the pixels in $D_{\text {norm }}$ and $d_{\text {rel }}$. As noted above there is a very large dispersion despite a good correlation factor. For $d_{\text {rel }}$ close to 0.5 , the $F W H M$ of the $D_{\text {norm }}$ distribution is of the order of 0.4 , while for $D_{\text {norm }}$ close to 0.5 the $F W H M$ of the $d_{\text {rel }}$ is also close to 0.4 . When considering the boundary $\left(d_{\text {rel }}\right.$ close to 1$)$, the $F W H M$ of the $D_{\text {norm }}$ distribution is similar. However it is much smaller (and no longer a Gaussian) when considering values of $D_{\text {norm }}$ close to 1 , as $d_{\text {rel }}$ peaks around 1 . 

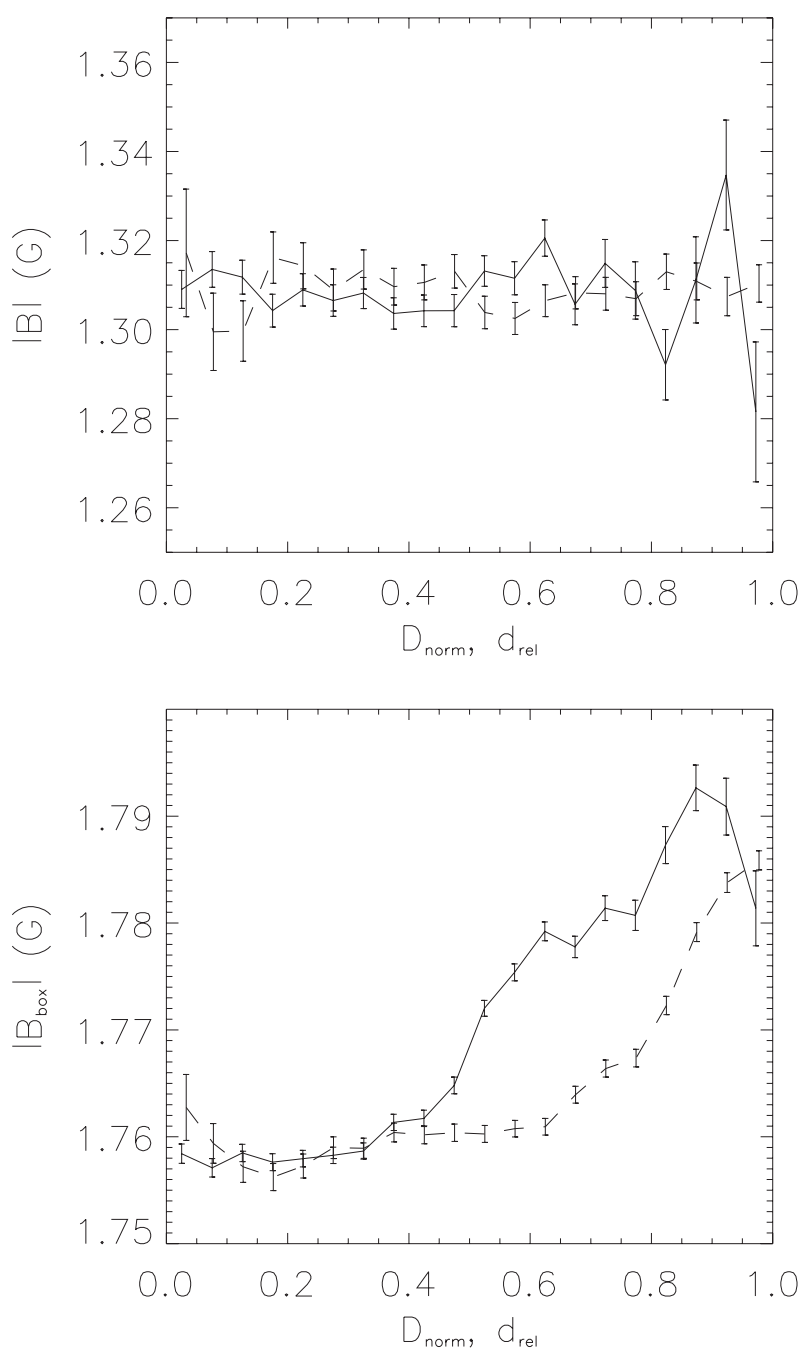

Fig. 8. Top panel: magnetic field $|B|$ in each pixel as a function of the normalized divergence $D_{\text {norm }}$ (solid line) and of the relative distance to cell center $d_{\text {rel }}$ (dashed line), for $N_{\text {box }}$ larger than 40 and $|B|<3 \mathrm{G}$. Lower panel: same for the magnetic field $\left|B_{\text {box }}\right|$ averaged over the $7 \times$ 7 pixel box.

\subsubsection{Magnetic field content}

It is important to check the magnetic field variations for this pixel selection. This is shown in Fig. 8. The variations of $|B|$ are very small and not significant, so at least the variations of $I$ are not expected to be affected by any residual magnetic field variations. Furthermore, the variation of $\left|B_{\text {box }}\right|$, affecting mostly $I_{\min }$ and $I_{\max }$, is significant but still very small, with a variation of the order of $0.03 \mathrm{G}$, while $\left|B_{\text {box }}\right|$ is always below $3.5 \mathrm{G}$. When looking at the variation of $I$ with $|B|$, we see that the variation in that domain of $|B|$ is very small, and such a small variation of $|B|$ would correspond to an intensity variation of 0.006 only (for an average level of $3930 \mathrm{ADU}$ ), which is negligible.

\subsubsection{Intensity variations}

We have defined a selection of pixels for which the influence of the magnetic field should be very small. We can now study the intensity variations inside supergranules. Figure 9 shows the intensity variations as a function of the normalized divergence $D_{\text {norm }}$ and of the relative distance to cell center $d_{\text {rel }}$, for $N_{\text {box }}$ larger than $40 . I$ decreases from the center of the cell to the boundary, either using $D_{\text {norm }}$ or $d_{\text {rel }} I_{\max }$ is also decreasing, while $I_{\min }$ is close to constant, leading to a smaller contrast at the cell boundary. Despite a consistent slope between the two approaches ( $D_{\text {norm }}$ and $\left.d_{\text {rel }}\right)$, all intensities increase again very close to the edge (converging flows) for $D_{\text {norm }}$ but decrease even more when using the geometrical approach. This may be due to the fact that even with a severe selection, there is still probably a small contamination from the network magnetic field in converging flows.

In order to quantify these variations, we have used two approaches. The first one is to compute the slope of these variations over the whole range. The results are shown in Table 1 . For $N_{\text {box }}$ larger than 40, the slopes are larger for $D_{\text {norm }}$ than for $d_{\text {rel }}$. Given their respective distribution, this means that the slope may be larger in the inner part of the cells. The slope for $I_{\min }$ is positive (showing an increase toward the boundary) for $D_{\text {norm }}$ but is not very significant, and it is not significantly different from zero for $d_{\text {rel }}$.

Another way to look at these variations is to consider what happens at the center and at the boundary. Here we compute the average intensities close to the cell center or maximum diverging flows (with $D_{\text {norm }}$ and $d_{\text {rel }}$ lower than 0.1 ), and close to the boundary or maximum converging flows (values above 0.9 ). Table 2 shows the intensity at the cell boundary minus the intensity at the cell center. Again for $N_{\text {box }}$ larger than 40, the signs are consistant with the slopes of Table 1 . However, the differences are now larger when using $d_{\text {rel }}$ than when using $D_{\text {norm }}$. This could be due to the observation made in Fig. 9 close to the boundary. This indicates that in that case, the variation may be reduced in the strong converging flows, but are strongly present everywhere else on the cell boundary.

\subsubsection{Variations for a more drastic selection}

Despite that the number of pixels available strongly decreases when a more severe selection is made using $N_{\text {box }}$, we also shows the results for $N_{\text {box }}$ larger than 44 . This leads to 89509 pixels, covering only $0.3 \%$ of the surface. The results are shown in Tables 1 and 2. In this case, both the slopes and the intensity differences are increased, even if the errobars become larger. Another important fact is that $I_{\min }$ then also decreases from the cell center to the boundary when considering $D_{\text {norm }}$. It is not significantly different from zero when considering $d_{\text {rel }}$.

\subsubsection{Discussion of errorbars and possible biases}

For $N_{\text {box }}$ larger than 40 for example, the slopes and intensity differences are very significant, with a S/N larger than 10 in many cases. So even if the errorbars are underestimated by a factor smaller than 2 as mentionned in Sect. 2.4, the results presented in this section remain very significant.

Because of the severe selection, it is important to consider possible biases that would affect the results. Indeed, we consider pixels with $|B|$ lower than $3 \mathrm{G}$ only. Therefore, in more active areas, we will select a larger proportion of pixels inside granules (i.e. where the intensity is the greatest) because the smallscale magnetic structures (unresolved here) are more likely to be located in intergranules (e.g. Domínguez Cerdeña et al. 2003). This should be the case closer to the boundary and to the magnetic network, and therefore we might expect an increase of intensity there due to the selection effect. Since we observe the opposite, this possible selection bias cannot be responsible for our results. 

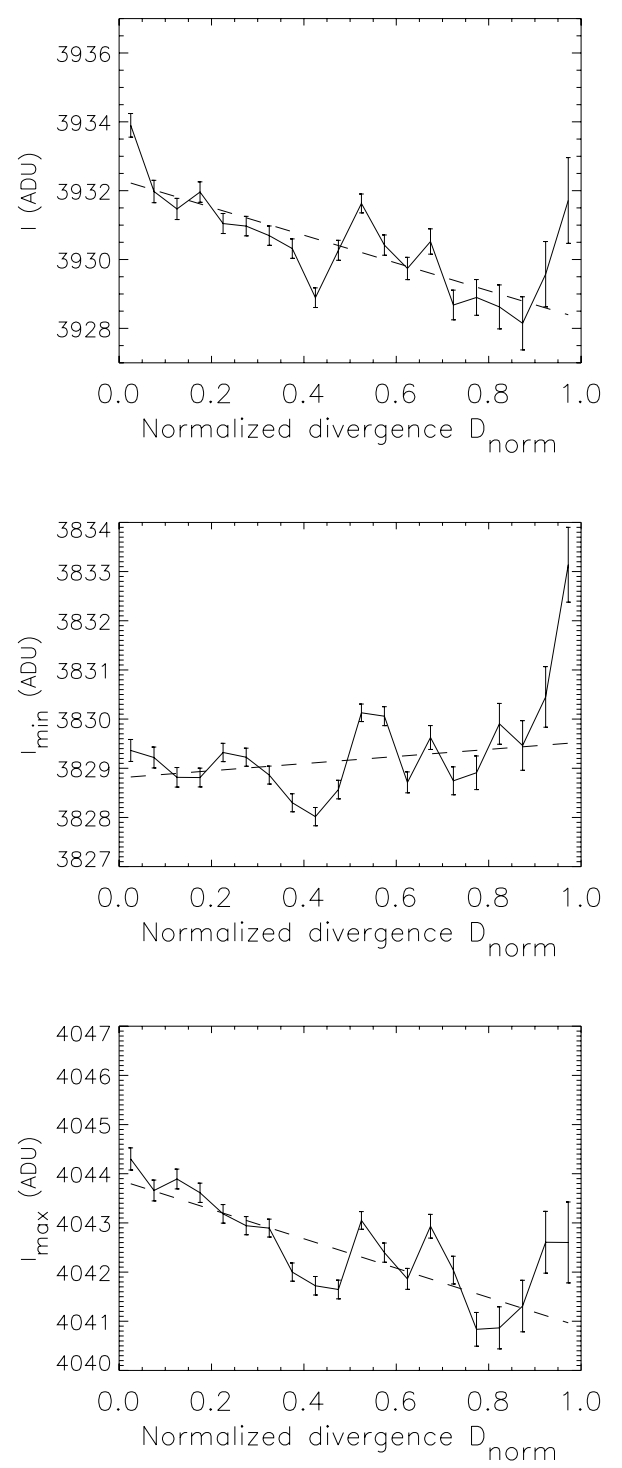
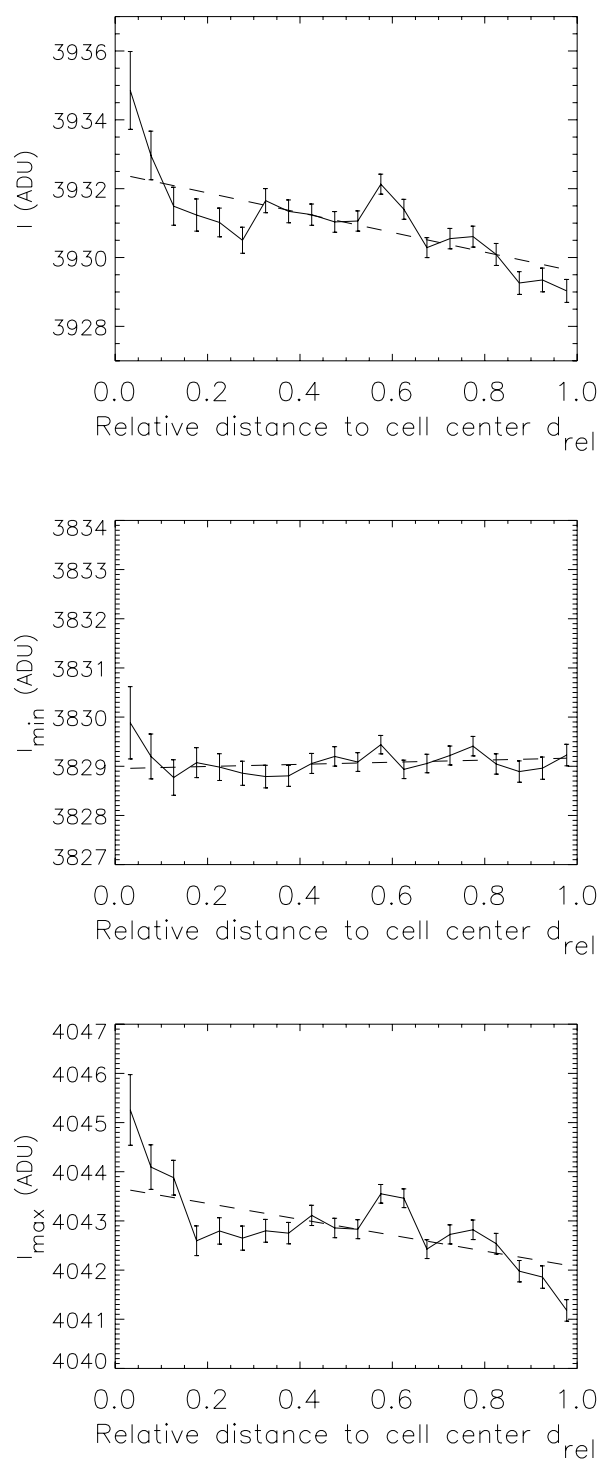

Fig. 9. Left panels: intensities versus the normalized divergence $D_{\text {norm }}$, for $N_{\text {box }}$ larger than 40 and $|B|<3 \mathrm{G}$. Right panels: same versus the relative distance to cell center $d_{\text {rel }}$. From top to bottom: intensity $I, I_{\min }$, and $I_{\max }$. The dashed lines are linear fits. Note that for a given variable, the scale is the same for $D_{\text {norm }}$ and $d_{\text {rel }}$ in order to make the comparison easier.
Table 1. Slopes of intensity $\left(I, I_{\min }, I_{\max }\right)$ versus the normalized divergence $D_{\text {norm }}$ and versus the relative distance to cell center $d_{\text {rel }}$, for $N_{\text {box }}$ larger than 40 (Cols. 2 and 3) and larger than 44 (Cols. 4 and 5) . Intensities are expressed in ADU and the average intensity is $3930 .|B|$ is smaller than $3 \mathrm{G}$.

\begin{tabular}{lcccc}
\hline \hline Int. & $D_{\text {norm }}, 40$ & $d_{\text {rel }}, 40$ & $D_{\text {norm }}, 44$ & $d_{\text {rel }}, 44$ \\
\hline$I$ & $-4.04 \pm 0.35$ & $-2.87 \pm 0.33$ & $-6.33 \pm 1.03$ & $-2.07 \pm 0.96$ \\
$I_{\min }$ & $0.72 \pm 0.23$ & $0.22 \pm 0.21$ & $-1.87 \pm 0.68$ & $-0.30 \pm 0.62$ \\
$I_{\max }$ & $-2.99 \pm 0.23$ & $-1.62 \pm 0.21$ & $-2.25 \pm 0.68$ & $-0.09 \pm 0.62$ \\
\hline
\end{tabular}

Decreasing further the threshold of $3 \mathrm{G}$ does not change the results, so the results are quite robust. Furthermore, we have performed simulations to better test the significance of the results. We choose random pixels in the maps that will be considered as artificial "cell centers". Then we use the same pixel selection as on the real data. We derive slopes showing the variation of the intensity between the cell center and the cell boundary: these slopes are more than one order of magnitude smaller than the observed slopes. Finally, we have also added a new selection by choosing only the cells for which the perimeter-area relation is not too far from a circle. The results are not significantly different either.
Table 2. Difference between intensities at the cell boundary and at the cell center (boundary intensity minus center intensity, see text), i.e. $I$, $I_{\min }, I_{\max }$, when using the normalized divergence $D_{\text {norm }}$ and the relative distance to cell center $d_{\text {rel }}$, for $N_{\text {box }}$ larger than 40 (Cols. 2 and 3) and larger than 44 (Cols. 4 and 5). Intensities are expressed in ADU and the average intensity is $3930 .|B|$ is smaller than $3 \mathrm{G}$.

\begin{tabular}{lcccc}
\hline \hline Int. & $D_{\text {norm }}, 40$ & $d_{\text {rel }}, 40$ & $D_{\text {norm }}, 44$ & $d_{\text {rel }}, 44$ \\
\hline$I$ & $-2.50 \pm 0.31$ & $-4.34 \pm 0.30$ & $-7.87 \pm 0.90$ & $-2.87 \pm 0.94$ \\
$I_{\min }$ & $2.27 \pm 0.20$ & $-0.25 \pm 0.19$ & $-2.60 \pm 0.61$ & $0.69 \pm 0.61$ \\
$I_{\max }$ & $-1.26 \pm 0.21$ & $-2.99 \pm 0.19$ & $-4.06 \pm 0.60$ & $-2.74 \pm 0.60$ \\
\hline
\end{tabular}

\subsection{Variation with cell size}

As we have a large sample of pixels, we attempted to study possible variations of the results with cell size. When considering various categories of sizes, we find that the results are quite constant, except for very large cells. However, there are only a few of these very large cells so the results are not significant in that domain. We conclude that at this point it is difficult to identify any significant variation with the cell size. However, this approach is very interesting because it also shows that the results are very robust when considering completely independant data sets of cells. 


\section{Discussion and conclusion}

When selecting the pixels associated with the lowest magnetic fields in order to avoid possible brightness enhancements due to the magnetic network, we observe a decrease of the intensity from the supergranule cell center to the boundary. The amplitude of the variation is of the order of 2-7 ADU depending on the more or less severe selection or on the type of computation (slope over the whole supergranule or difference between the intensities at the center and boundary), which corresponds to a variation of $0.05 \%$ to $0.18 \%$ in brightness. If we consider an average temperature of $5800 \mathrm{~K}$, this corresponds to a significant variation in temperature in the range $0.8-2.8 \mathrm{~K}$. This can be compared to the upper limit of $2-3 \mathrm{~K}$ on non-axisymmetric brightness inhomogeneities over scales between $5 \times 10^{4}$ and $2 \times 10^{5} \mathrm{~km}$ (i.e. in a range above the supergranule scale) found by Foukal \& Fowler (1984). Furthermore, our results are very stable with respect to the selection, and there is a systematic increase of the intensity variation when the selection is more drastic. We have also noted that the variations are quite sharp at the center of the cells.

A new result has also been obtained concerning the variation of the intensity as a function of the magnetic field, as we observe a dependence on the localisation in the cell. Network magnetic fields are associated with a larger intensity close to the cell center compared to those close to the boundary, while it is the opposite for lower magnetic field structures, which are probably related to intranetwork magnetic fields.

Our work also examines in more detail the intensity variation across the supergranules. Is this variation the same everywhere? Is it different for granules and intergranules? To answer these questions we have also considered the minimum intensity (intergranule) and maximum intensity (granule center) in boxes around each pixel. We find that the behavior of the granules is close to the general variation, but tends to exhibit smaller slopes. On the other hand, the intergranules show a decrease from cell center to boundary only when the selection is very restrictive: in all cases the slope is very small.

It could be argued that the observed intensity variation could be due to a different size distribution of granules at the center of supergranules and at the boundary. For example, Brandt et al. (1991) found an excess of small granules in converging flows at the mesogranulation scale. As the magnetic field tends to be larger at the boundary of supergranule cells, we expect to have smaller granules (Hanslmeier et al. 1991; Hirzberger et al. 1999). However, Hirzberger et al. (1997) have observed that when the granule size increases, the maximum intensity also increases but the minimum intensity decreases. Granulation simulations (Gadun et al. 2000) confirm the observations of Hirzberger et al. (1997). So if the intensity variation is due to this size effect, we expect to observe a decrease in intensity and maximum intensity (which we do observe) and an increase in minimum intensity, which we do not observe (we find an almost constant minimum intensity, or a decreasing one).

We conclude that the intensity variations expected from smaller granules at the cell boundary are not sufficient to explain our results. It reinforces the possibility of a lower average temperature at the boundary of supergranules. A lower temperature at the boundary is compatible with a convective nature of solar granulation. However, an open question is now whether this intensity variation in granules and intergranules is the one that would be observed if one decreases the average temperature of region by a given amount, or if the behavior of intergranules and granules is expected to be different. Furthermore, the intensity profiles close to the cell center do not seem be flat as is the case for granules.

Comparison with large-scale simulations of the solar surface such as those performed by Rieutord et al. (2002) and Benson et al. (2006) will therefore be performed order to check that the observed profiles and intergranule and granule properties are compatible with convection. It will also have to be checked with higher spatial resolution observations on a large field-of-view, such as those that will soon be obtained with CALAS ${ }^{1}$ at the Pic du Midi Observatory (Meunier et al. 2003, 2005). This should improve the measurements of granules and intergranules.

Acknowledgements. We thank M. Rieutord for useful comments on the manuscript. SOHO is a mission of international cooperation between the European Space Agency (ESA) and NASA.

\section{References}

Beckers, J. M. 1968, Sol. Phys., 5, 309

Benson, D., Stein, R. F., \& Nordlund, A. 2006, AAS/Solar Physics Division Meeting, 37, 30.03

Brandt, P., Ferguson, S., Scharmer, G., et al. 1991, A\&A, 241, 219

Domínguez Cerdeña, I., Sánchez Almeida, J., \& Kneer, F. 2003, A\&A, 407, 741

Foukal, P., \& Fowler, L. 1984, ApJ., 281, 442

Frazier, E. N. 1970, Sol. Phys., 14, 89

Gadun, A. S., Hanslmeier, A., Pikalov, K. N., et al. 2000, A\&AS, 146, 267

Hagenaar, H. J., Schrijver, C. J., \& Title, A. M. 1997, ApJ., 481, 988

Hanslmeier, A., Nesis, A., \& Mattig, W. 1991, A\&A, 251, 307

Hart, A. B. 1954, MNRAS, 114, 17

Hirzberger, J., Bonet, J. A., Vázquez, M., \& Hanslmeier, A. 1999, ApJ., 515, 441

Hirzberger, J., Vázquez, M., Bonet, J. A., Hanslmeier, A., \& Sobotka, M. 1997, ApJ., 480, 406

Koutchmy, S., \& Lebecq, C. 1986, A\&A, 169, 323

Lin, H., \& Kuhn, J. R. 1992, Sol. Phys., 141, 1

Meunier, N., Rieutord, M., \& Beigbeder, F. 2003, in SF2A-2003: Semaine de 1'Astrophysique Francaise, ed. F. Combes, D. Barret, T. Contini, \& L. Pagani, 93

Meunier, N., Rondi, S., Tkaczuk, R., Rieutord, M., \& Beigbeder, F. 2005, in Large-scale Structures and their Role in Solar Activity, ed. K. Sankarasubramanian, M. Penn, \& A. Pevtsov, ASP Conf. Ser. 346, 53

Meunier, N., Tkaczuk, R., Roudier, T., \& Rieutord, M. 2007, A\&A, 461, 1141 Montagne, M., Muller, R., \& Vigneau, J. 1996, A\&A, 311, 304

Rast, M. P. 2003a, in GONG+ 2002. Local and Global Helioseismology: the Present and Future, ed. H. Sawaya-Lacoste, ESA SP-517: 163

Rast, M. P. 2003b, ApJ., 597, 1200

Rieutord, M., Ludwig, H.-G., Roudier, T., Nordlund, A., \& Stein, R. 2002, Nuovo Cimento C Geophysics Space Physics C, 25, 523

Rieutord, M., Roudier, T., Malherbe, J. M., \& Rincon, F. 2000, A\&A, 357, 1063

Scherrer, P. H., Bogart, R. S., Bush, R. I., et al. 1995, Sol. Phys., 162, 129

Simon, G., \& Leighton, R. 1964, ApJ., 140, 1120

Title, A. M., Tarbell, T. D., \& Topka, K. P. 1987, ApJ, 317, 892

Title, A. M., Topka, K. P., Tarbell, T. D., et al. 1992, ApJ, 393, 782

van der Borght, R. 1979, MNRAS, 188, 615

Wang, H. 1988, Sol. Phys., 117, 343 